\title{
Recommendation for a set of solar EUV lines to be monitored for aeronomy applications
}

\author{
J. Lilensten ${ }^{1}$, T. Dudok de Wit ${ }^{2}$, P.-O. Amblard ${ }^{3}$, J. Aboudarham ${ }^{4}$, F. Auchère ${ }^{5}$, and M. Kretzschmar ${ }^{6}$ \\ ${ }^{1}$ LPG, CNRS and Joseph Fourier University, Bâtiment D de Physique, BP 53, 38041 Saint-Martin d'Hères cedex 9, France \\ ${ }^{2}$ LPCE, CNRS and University of Orléans, 3A Avenue de la Recherche Scientifique, 45071 Orléans cedex 2, France \\ ${ }^{3}$ LIS, CNRS, 961 Rue de la Houille Blanche, BP 46, 38402 St. Martin d'Hères cedex, France \\ ${ }^{4}$ LESIA, Paris Observatory, 5 Place Jules Janssen, 92195 Meudon, France \\ ${ }^{5}$ IAS, CNRS and Paris-Sud University, Bt. 121, 92405 Orsay cedex, France \\ ${ }^{6}$ SIDC, Royal Observatory of Belgium, Avenue Circulaire 3, 1180 Brussels, Belgium
}

Received: 7 November 2006 - Revised: 15 April 2007 - Accepted: 7 May 2007 - Published: 29 June 2007

\begin{abstract}
In two recent studies, Dudok de Wit et al. (2005) and Kretzschmar et al. (2006) have shown that the solar Ultra-Violet spectrum between 25 and $195 \mathrm{~nm}$ can be reconstructed from the observation of a set of 6 to 10 carefully chosen spectral lines. The best set of lines, however, is application dependent. In this study, we demonstrate that a good candidate for aeronomy applications consists of the following 6 lines: $\mathrm{H}$ I at $102.572 \mathrm{~nm}, \mathrm{C}$ III at $97.702 \mathrm{~nm}, \mathrm{O} \mathrm{V}$ at $62.973 \mathrm{~nm}, \mathrm{He} \mathrm{I}$ at $58.433 \mathrm{~nm}, \mathrm{Fe} \mathrm{XV}$ at $28.415 \mathrm{~nm}$ and $\mathrm{He}$ II at $30.378 \mathrm{~nm}$. The TRANSCAR model is used to quantify the impact of each individual line on the density, temperature and velocity profiles. Using a multidimensional scaling technique, we show how to select from this the best set of lines. Although this selection is motivated by the specification of the ionosphere, our set of lines is also found to be appropriate for reconstructing the variability of the solar spectrum between 25 and $195 \mathrm{~nm}$.
\end{abstract}

Keywords. Ionosphere (Modeling and forecasting; Solar radiation and cosmic ray effects) - Solar physics, astrophysics, and astronomy (Ultraviolet emissions)

\section{Introduction}

The continuous monitoring and the real-time estimation of the solar irradiance in the X-ray (XUV, from 1 to $10 \mathrm{~nm}$ ) and Extreme Ultraviolet (EUV, from 10 to $121 \mathrm{~nm}$ ) spectral bands is a crucial issue in space weather. The prime application is orbitography.The XUV/EUV flux is the main energy input for thermosphere/ionosphere system. Sudden increases

Correspondence to: J. Lilensten

(jean.lilensten@obs.ujf-grenoble.fr) in the flux heat the thermosphere, cause it to expand, which, in turn, increases the friction for low orbit spacecraft. The consequence is altitude loss and orbital acceleration. The specification of the thermosphere is also needed for the localization of space debris. A second important group of users is telecommunications and positioning, including groundspace and space to space communications. The ionosphere acts as a filter for electromagnetic waves, so that sudden changes in solar activity also result in a degradation of the positioning and perturbations (or even loss) of communications.

The solar XUV/EUV flux is very variable and is primarily governed by solar magnetic activity.

- Short-term variations (on scales of minutes to hours) are related to solar eruptive phenomena.

- Intermediate term variations, modulated by the 27-day rotation period of the Sun, are related to the appearance and disappearance of active regions, plages, network and coronal holes on the solar disk.

- Long-term variability is related to the 22-year magnetic cycle of the Sun.

As of today, there has been no permanent monitoring of the solar XUV/EUV irradiance with sufficient radiometric accuracy $(<10 \%)$ and over more than one solar cycle. Most missions have either provided high spectral resolution with little temporal coverage, or the converse. This has resulted in a large EUV hole (Woods et al., 2005) that has only ended in 2002.

Spectrophotometric satellite XUV/EUV observations started in the 1960s and daily observations were made by Atmospheric Explorer-E until 1981 (Hinteregger, 1981).

Published by Copernicus Publications on behalf of the European Geosciences Union. 
Since that time, however, there have only been a few occasional solar XUV/EUV irradiance measurements, such as the San Marco 5 satellite (Schmidtke et al., 1992; Tobiska et al., 1993) and several sounding rockets. Moreover, for wavelengths short of $110 \mathrm{~nm}$, no space-borne spectrograph has been calibrated in-flight. Continuous measurements have started again in the 1990s with the Solar EUV Monitor (SEM) on board SOHO (Judge et al., 1998), the Upper Atmosphere Research Satellite (UARS; Woods et al., 1996) and the Solar Radiation \& Climate Experiment (SORCE; Rottman, 2005). Unfortunately, these measurements are of little use for aeronomy, as they are either broadband (SEM) or do not properly cover the EUV range (SEM and UARS).

Due to the lack of adequate irradiance data, most thermosphere/ionosphere models rely today on proxies for the solar irradiance, such as the $f_{10.7}$ index (radio flux at $10.7 \mathrm{~cm}$ ) or the magnesium line Mg II K (Heath and Schlesinger, 1986). The solar radio emission at $10.7 \mathrm{~cm}$ is generated by a variety of physical processes that occur from the solar corona down to the photosphere. Although this parameter correlates reasonably well with the solar XUV/EUV flux, it clearly cannot replace it. Several other proxies have been developed, but none of them can be used as a substitute for the irradiance (Floyd et al., 2005).

Continuous and spectrally resolved measurements of the solar EUV irradiance resumed in February 2002 with the Solar EUV Experiment (SEE) grating spectrograph on board the TIMED satellite (Woods et al., 2005). The prime science objective of the TIMED mission is to understand the energy transfer into and out of the Mesosphere and Lower Thermosphere/Ionosphere (MLTI) region of the Earth's atmosphere, and the basic structures that result from the energy transfer into these regions. The SEE suite consists of a spectrograph and a series of photometers designed to measure solar ultraviolet radiation from 0.1 to $194 \mathrm{~nm}$.

Using a statistical analysis of 2 years of SEE data, Dudok de Wit et al. (2005) have shown that it is possible to reconstruct the full XUV/EUV spectrum from a linear combination of 6 to 10 spectral lines only, with an accuracy better than $5 \%$. It is interesting to note that the same small sets of lines had been identified earlier by using a different method. Kretzschmar et al. (2004) used a broad set of optically thin lines measured by the SUMER (Solar Ultraviolet Measurements of Emitted Radiation) instrument on board SOHO to compute a mean Differential Emission Measure (DEM), which was then inverted. This resulted in a reference spectrum for the quiet Sun. Using this physical approach, Kretzschmar et al. (2006) were able to retrieve the whole irradiance spectrum from the observation of 6 reference lines.

In both approaches, the choice of the most appropriate lines is application dependent. For aeronomy, the prime objective is the specification of the thermosphere/ionosphere system, whereas in solar physics, the reconstruction of the spectrum matters more. Dudok de Wit et al. (2005) provide a methodology for selecting the best set of lines; here we use the same approach to determine which set of lines is most appropriate for aeronomy purposes. Due to the lack of observational data, we use the TRANSCAR ionospheric model (Diloy et al., 1996) to quantify the impact of each individual spectral line on the plasma parameters. We only consider the EUV range, since this has the strongest impact on the thermosphere/ionosphere system. The solar irradiance in the XUV and UV is also of importance for space weather (Danilov, 2006), impacting, for example, the ozone concentration in the lower mesosphere and in the upper stratosphere (Rozanov et al., 2005). These effects, however, occur at altitudes far below the ones we are interested in (typically between 100 and $800 \mathrm{~km}$ ), in which satellite drag is an important issue.

\section{Description of the method}

Particle populations in the ionosphere can be conveniently split into a large thermal Maxwellian population and a small non-thermal one. Each ion species, however, has its own distribution, which differs from that of the electrons. The processes which drive these populations are strongly timedependent, as they are governed by chemical reactions, by collision frequencies, by the dynamics (neutral winds), etc; a time-dependant fluid description is required to properly describe the behaviour of these populations.

The hottest population mainly consists of electrons with non-Maxwellian distributions, whose energy exceeds the mean thermal energy by orders of magnitude. Their energy source is located either in the magnetosphere (electron and proton precipitations) or in the atmosphere (photoionization). Each primary particle can cause many ionizations and excitations. Transport, however, is fast enough to be considered as instantaneous versus the characteristic time scale of the precipitation event or the solar event. This means that nonthermal particles can be adequately described by a steadystate kinetic description.

The TRANSCAR model (TRANSCAR stands for "TRANSport au CARré", or "squared transport") precisely provides such a two-population description of the ionosphere (Diloy et al., 1996); it has been shown to describe the ionosphere both from a statistical point of view and for specific events (Lilensten et al., 1996; Blelly et al., 1996; Witasse et al., 1999; Lilensten and Bornarel, 2005). TRANSCAR describes the non-thermal population by a kinetic Boltzmann equation (Lummerzheim and Lilensten, 1994) for which the $\mathrm{XUV/EUV} \mathrm{solar} \mathrm{flux} \mathrm{and} \mathrm{particle} \mathrm{precipitation} \mathrm{are} \mathrm{given} \mathrm{as}$ inputs. The non-thermal particles interact with the neutral atmosphere (Picone et al., 2002), taking into account the absorption and the collision cross sections of each neutral constituent. Other inputs are the electron density and temperature profiles, which are computed by the fluid part of the model. The kinetic part of the model estimates (together with other parameters) the ion and electron production and 
the heating rates, which, in turn, feed the fluid part of the model.

The fluid part of TRANSCAR is based on an eighthmoment approximation of Boltzmann's equation (Schunk and Nagy, 2004). The resulting set of equations, projected along the direction of the magnetic field, allows one to determine the density, velocity, temperature and heat flow for each ion species. Electron density and velocity profiles are estimated assuming charge neutrality and ambipolar flow, while taking into account the effect of the electric field. TRANSCAR describes the ionosphere from 100 up to $3000 \mathrm{~km}$, solving the set of equations for molecular and for atomic ions. In this paper, we restrict our study to altitudes below $800 \mathrm{~km}$, where most of the production by photoionization occurs.

To summarize, the main inputs we use here are the spectrally resolved EUV flux and the neutral atmosphere, while the main outputs are the electron and ion production rates, concentrations, temperatures and velocity profiles between 100 and $800 \mathrm{~km}$.

\section{Description of the XUV/EUV flux}

Most of the current EUV / XUV models rely on a few experiments made by the Atmospheric Explorer missions (Hinteregger, 1981) and by rocket flights. Hinteregger (1981) and Hinteregger and Katsura (1981) gave a first representation of solar XUV/EUV fluxes for aeronomical applications. A first reference flux SC\#21REF was assembled from measurements performed in July $1976\left(f_{10.7}=70\right)$, and given in 1659 wavelengths. Torr and Torr (1979) and Torr and Torr (1985) later proposed two reference fluxes for aeronomy called F79050N $\left(f_{10.7}=243\right)$ and SC\#21REF $\left(f_{10.7}=68\right)$. Their UV spectrum was divided into 39 bins, some of which correspond to intense spectral lines: $\mathrm{He}$ II and Si X at $25.63 \mathrm{~nm}, \mathrm{Fe} X \mathrm{XV}$ at $28.415 \mathrm{~nm}, \mathrm{Si} \mathrm{XI}$ at $30.331 \mathrm{~nm}$, He II at $30.378 \mathrm{~nm}, \mathrm{Mg}$ IX at $36.807 \mathrm{~nm}$, Ne VII at $46.522 \mathrm{~nm}$, OVI at $55.437 \mathrm{~nm}, \mathrm{He} I$ at $58.433 \mathrm{~nm}, \mathrm{Mg} \mathrm{X}$ at $60.976 \mathrm{~nm}, \mathrm{O}$ V at $62.973 \mathrm{~nm}, \mathrm{O}$ III at $70.331 \mathrm{~nm}, \mathrm{~N}$ IV at $76.515 \mathrm{~nm}$, Ne VIII at $77.041 \mathrm{~nm}, \mathrm{O}$ IV at $78.936 \mathrm{~nm}, \mathrm{C}$ III at $97.702 \mathrm{~nm}, \mathrm{H} \mathrm{I}$ at $102.572 \mathrm{~nm}$ (Lyman $\alpha$ ) and finally O VI at $103.191 \mathrm{~nm}$. The Ly $\alpha$ line at $121.565 \mathrm{~nm}$ does not appear in this list because it is not energetic enough to ionise the terrestrial thermosphere. For terrestrial aeronomy, O VI is not energetic enough either, which leaves us with 16 pertinent lines only. The remaining boxes represent the mixing of close lines and the continuum. This work turned out to be extremely useful, partly because the authors proposed the corresponding absorption and ionisation cross sections for the major thermospheric species. Most of the current aeronomic calculations are still based on this set of 39 lines, whose wavelengths (respectively energies) range from $2.37 \mathrm{~nm}(532 \mathrm{eV})$ to $103.191 \mathrm{~nm}(12.015 \mathrm{eV})$, thus covering the XUV and EUV parts of the solar spectrum.
The above-mentioned reference fluxes have proven to be sufficiently accurate for most applications and so existing models are very unlikely to be modified in a near future to take into account other lines. For that reason, we shall also restrict ourselves here to the 16 most intense lines of this set. One of the questions to be addressed below is whether this set of lines is sufficiently informative, i.e. whether the full $\mathrm{XUV/EUV} \mathrm{spectrum} \mathrm{can} \mathrm{be} \mathrm{reconstructed} \mathrm{from} \mathrm{it.}$

Several authors have developed new models to take better advantage of the AE database. Tobiska (1991) and Tobiska and Eparvier (1998) developed a model called EUV, which takes data from satellites, rockets and ground-based facilities. The more recent SOLAR2000 model provides several indices that are tailored to specific needs, such as the S10.7 index for the specification of the neutral density (Tobiska and Nusinov, 2006). These quantities, however, may not be considered as indices, since they result from a computation and not from direct observations (Lathuillère and Menvielle, 2004). Another improved model is EUVAC (EUV for Aeronomic Calculations) (Richards et al., 1994), which differs from the previous ones by the choice of the reference flux, and the interpolation formula.

For the present study, we use Torr and Torr's model, as its dependence on solar activity is governed by $f_{10.7}$ only and not the date. To emphasize the effects of the lines, we use the reference flux F79050N, which corresponds to a very active Sun $\left(f_{10.7}=243\right)$. Wavelengths greater than $103.191 \mathrm{~nm}$ (and especially Ly $\alpha$ at $121.565 \mathrm{~nm}$ ) are not considered here, since they cannot ionize from the ground. Such wavelengths are nevertheless important because they dissociate heat the thermosphere. The ion densities are affected by the Ly $\alpha$ line and the continuum above it because of the molecule destruction. The temperature is affected because of heating. For all these effects, however, have been compared with our calculations using various instruments. We compared ion/electron densities and temperatures (Diloy et al., 1996) and conductivities (Lilensten et al., 1996) with the EISCAT incoherent scatter radar; emission rates were investigated with optical facilities (Witasse et al., 1999) and in different geomagnetic conditions (Blelly et al., 2005). All these comparisons, the impact falls within the instrumental error bars. Although this is not a reason to exclude longer wavelengths in the future, it justifies the choice of a reduced spectral range in the present study.

Let us also mention that the Ly $\alpha$ line at $102.5 \mathrm{~nm}$ can ionize NO and all the neutral mesospheric constituents, and that the wavelengths short of about $2 \mathrm{~nm}$ have an important impact on the $\mathrm{D}$ region. These effects should be taken into account in the study of altitudes below $100 \mathrm{~km}$.

\section{Effect of the spectrum on the ionosphere}

The main outputs of TRANSCAR are shown in Fig. 1 for solar minimum conditions. Quiet conditions are chosen in 


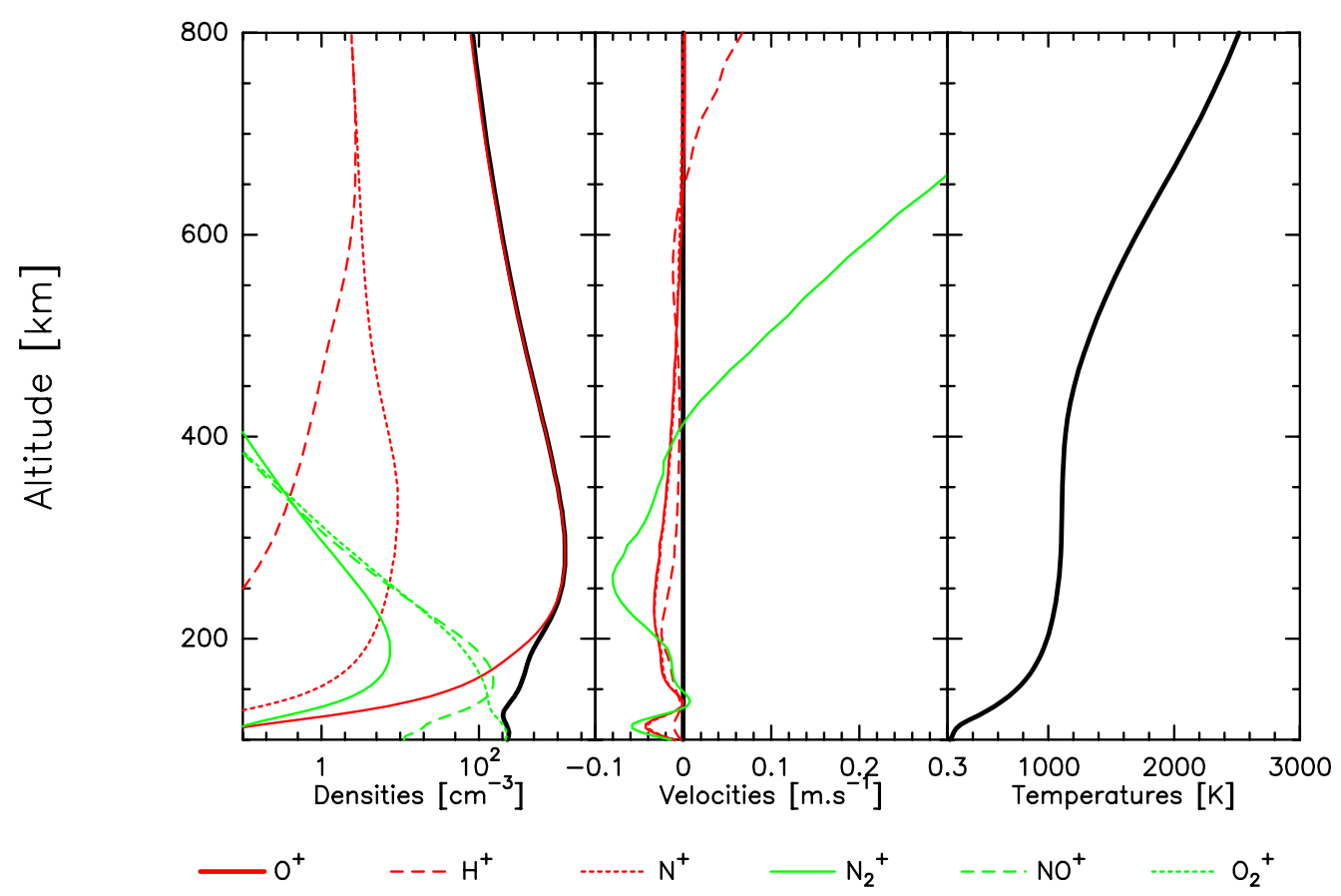

Fig. 1. State of the ionosphere under quiet conditions, as obtained from the TRANSCAR model with: the ion density profile (left panel, the black curve refers to the total density), the ion velocity profile (middle panel) and the electron temperature profile (right panel).

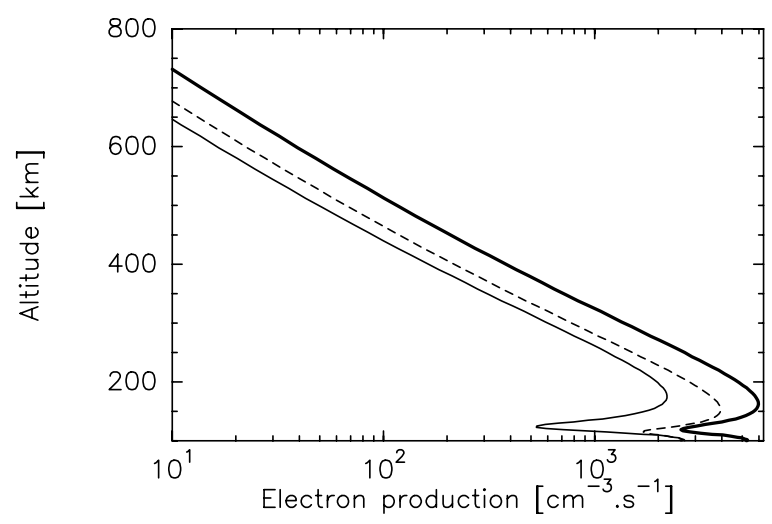

Fig. 2. Electron production rate associated with the continuum (dashed line) and the set of 16 spectral lines used in XUV/EUV aeronomical models (thin full line). The total production is shown in a thick full line.

order to focus on the effect on the production rates and the densities. This choice does not have strong implications on our conclusions. The relative intensity of the lines does, of course, vary in time (Schwenn, 2006), as coronal emissions are more strongly modulated by the solar cycle than chromospheric emissions. The altitude, however, where the energy is deposited, does not change drastically, and the ratios between chromospheric and coronal solar lines do not vary enough to invert our conclusions.
We model the electron production rate at mid-latitude $\left(45^{\circ}\right.$ geographic latitude), at local noon for day 180 (i.e. midJune). The $\mathrm{F}$ region peaks at $280 \mathrm{~km}$, with $\mathrm{O}+$ being as usual the dominant ion in that region and $\mathrm{NO}+/ \mathrm{O} 2+$ the dominant ones in the lower $\mathrm{F}$ and $\mathrm{E}$ regions.

The ion velocities are shown in the middle panel of Fig. 1 . There is a slight downward drift below $400 \mathrm{~km}$, but the values remain very small. Since we want to focus on the effect of the solar flux, we do not consider here any electric field or particle precipitation. The ionosphere then remains quiet, with vertical velocities much below $1 \mathrm{~m} / \mathrm{s}$. Above approximately $500 \mathrm{~km}$, the molecular ion velocity increases but the molecular ion density remains very low. This density corresponds to a diffusion of the molecular ions inside the $\mathrm{O}+$ population. The velocity peaks around $800 \mathrm{~km}$ and strongly drops above (not shown here) (Lilensten and Blelly, 2000). The electron temperature profile (right panel of Fig. 1) increases above about $400 \mathrm{~km}$ due to the high XUV/EUV flux.

\subsection{Effect of the continuum on the electron production}

"Continuum" refers here to the XUV/EUV spectrum, except for the set of strong 16 spectral lines that are commonly used in aeronomical models. This definition, which is frequently used in aeronomy, differs from the one used in solar physics. The electron production profiles associated with the continuum and the 16 spectral lines are shown in Fig. 2. 
The continuum accounts for approximately one half of the total production at low altitude (below $125 \mathrm{~km}$ ) and slightly more above; neither the continuum nor the spectral lines may therefore be neglected.

\subsection{Effect of the different lines on the electron production}

To determine the electron production associated with each of the 16 spectral lines, we simply run the kinetic part of TRANSCAR with each single line turned on individually and the rest of the spectrum, including the continuum, turned off. In doing so, we can precisely determine where the energy is deposited. Figure 3 shows the electron production rate associated with each line, while Fig. 4 displays the production ratio.

From Fig. 4, we can see that He II contributes to about $20 \%$ of the total electron production above about $150 \mathrm{~km}$, while H I and C III produce, respectively, about $40 \%$ at $100 \mathrm{~km}$ and $20 \%$ at $120 \mathrm{~km}$. These 3 lines are the most important ones for monitoring the electron production.

\subsection{Effect of the different lines on densities and tempera- tures}

While the electron production rate can easily be determined by turning on each spectral line individually, this procedure does not apply anymore for macroscopic parameters. Indeed, if we turn on a single line, a thin slab will be ionized and heated, creating large and totally unrealistic temperature and production gradients. The system then becomes discontinuous and the differential fluid transport equations cannot be solved anymore. Therefore, to study the effect of a given wavelength $\lambda$ on an ionospheric parameter $X$ (density, temperature or velocity), we do the opposite and turn on all but one spectral line. Next we compute the relative contribution of that spectral line as a function of altitude $h$

$R_{\lambda}(h)=\frac{X_{\text {full }}(h)-X_{\lambda}(h)}{X_{\text {full }}(h)}$,

where $X_{\text {full }}(h)$ is the result obtained with the full Sun conditions (i.e. no lines turned off), and $X_{\lambda}(h)$ the result obtained with line $\lambda$ turned off. This method supposes that the contributions of the lines are weak enough for the effects to be linear. To check this, we successively computed the relative contribution $R_{\lambda}(h)$ for two intense lines: He II $(30.378 \mathrm{~nm})$ and C III $(97.702 \mathrm{~nm})$. These lines were first turned on and then turned off simultaneously. The impact on the production rate is illustrated in Fig. 5, which shows the production rate. We indeed find that the production rate of the sum equals the sum of the production rates. The discrepancy is largest around $150 \mathrm{~km}$, where it remains below 3\%; the resolution of the plot does not allow one to see it. We can therefore safely assume that the production rate is linear in the parameters versus the wavelengths. The same conclusion holds for the density and the temperature.
The relative contribution of each spectral line to the electron density, the electron temperature and the velocity are, respectively, displayed in Figs. 6 to 8. The H I lines produce about $30 \%$ of the electrons around $100 \mathrm{~km}$ but have very little impact on the temperature or on the dynamics, see Fig. 6. The C III line is the most important one around $110 \mathrm{~km}$. H I, together with C III, are key lines for monitoring the $\mathrm{E}$ and lower F regions. He II is the main production line at $150 \mathrm{~km}$, and is also a dominant source of energy for the electron density at higher altitudes. $\mathrm{O} \mathrm{V}$ is acting very efficiently above $200 \mathrm{~km}$, as well as He I. Note, however, that several lines lead to similar profiles. The deposition profiles of $\mathrm{Fe} \mathrm{XV}$ and $\mathrm{Si}$ $\mathrm{XI}$, for example, are very similar in shape. The impacts of $\mathrm{O} \mathrm{V}, \mathrm{Fe} \mathrm{XV}$ and $\mathrm{Si} \mathrm{XI}$ on the density are much alike, as are the temperature responses to Fe XV and Si XI. In the next section, we shall provide a method for eliminating lines that have redundant signatures.

The lines that mostly impact the temperature (see Fig. 7) are again $\mathrm{He}$ II, but also Fe XV and Si XI. These are also the most intense amongst the energetic lines. Note that the sum of all the contributions is not equal to one. Several phenomena influence the temperature of the ionosphere through various energetic couplings: frictional heating of the atmosphere, thermalisation of the photoelectrons, energy transfer by thermalisation of the precipitating electrons, and energy input by a downward electron heat flow. Frictional electron heating is negligible because of the small electron mass. The main source of energy comes from suprathermal electrons. The energy transfer to thermal electrons (Swartz and Nisbet, 1972) is particularly efficient at low energies, typically below $10 \mathrm{eV}$. To obtain these low energy electrons to heat the thermal bulk, it is necessary either to ionize the neutrals with energies close to the ionization threshold (typically below $22 \mathrm{eV}$ or wavelengths above $55 \mathrm{~nm}$ ), or to produce many energetic primary electrons. In the first case, the primary electrons have little energy, as they are created with the energy of the incident photon minus the ionization threshold (about $13 \mathrm{eV}$ ). At these energies, however, the excitation is also very efficient; such photons can generate several electrons, thus producing some electron heating. In this aspect, the continuum heats the ionosphere more efficiently than the lines. In the second case (with high energy photons), ionization is much more efficient than excitation. Many primary electrons are generated, which can produce secondary electons of any energy (including low ones) through collisions with neutrals. Secondary electrons with energies typically below $10 \mathrm{eV}$ start heating the ambient thermal electrons. At higher energies, most of the solar irradiance is already contained in the continuum of the current models. This explains the results shown in Fig. 7.

Besides heating, one must also include losses, as these can be important in the $\mathrm{E}$ and $\mathrm{F}$ regions. These losses correspond to inelastic collisions processes. The electron gas collides with the neutral atmosphere and can then excite either the rotational or vibrational states of the molecular species N2 


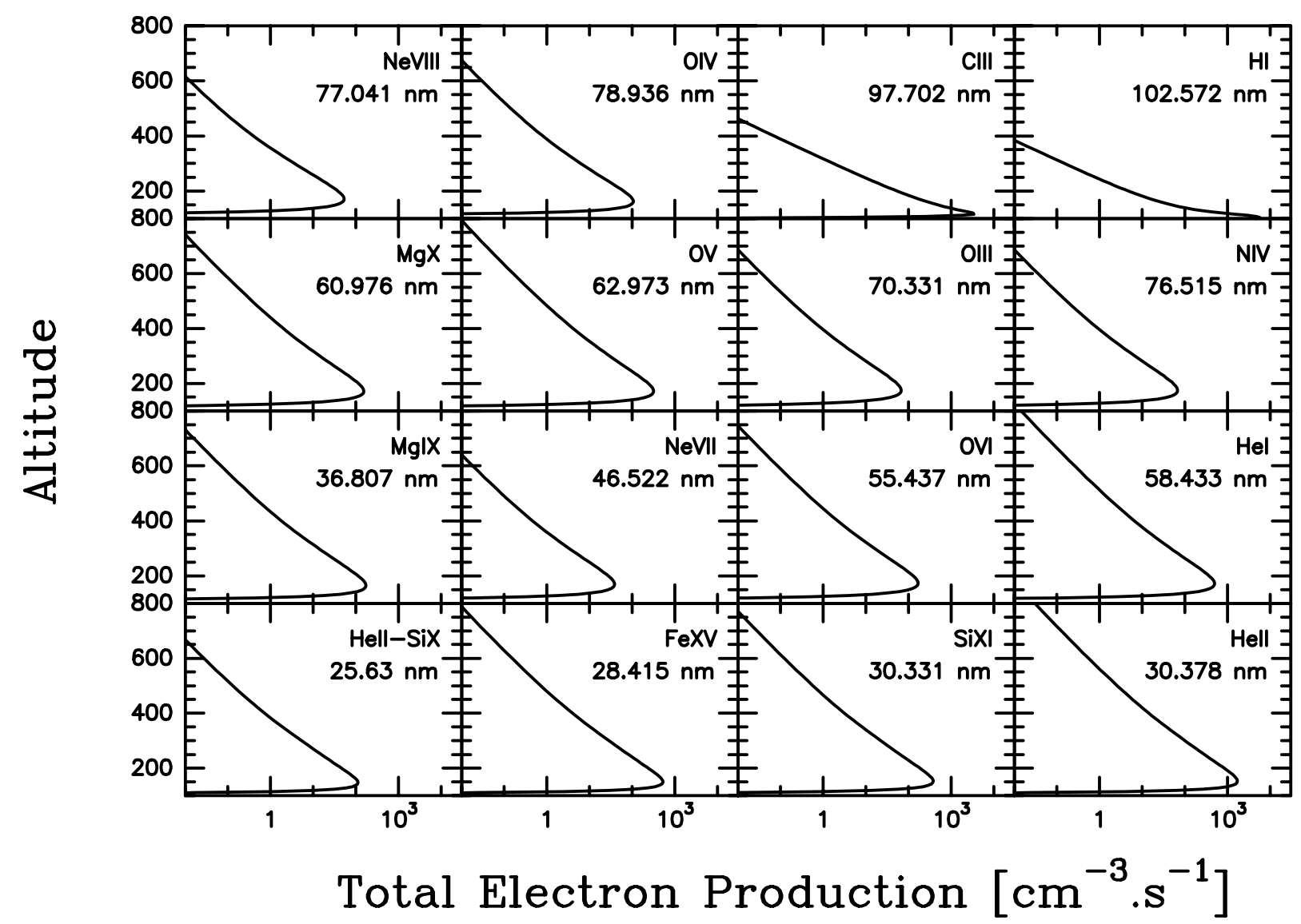

Fig. 3. Electron production rate associated with each of the 16 spectral lines versus altitude in $\mathrm{km}$.

and $\mathrm{O} 2$, or the atomic oxygen to the 1-D state, or the fine structure levels of $\mathrm{O}$. These processes are detailed in the book by Schunk and Nagy (2004).

The effect of the solar XUV/EUV on the velocities is not shown here. The reason is as explained above: the regular $\mathrm{XUV/EUV} \mathrm{flux} \mathrm{is} \mathrm{not} \mathrm{the} \mathrm{main} \mathrm{driver} \mathrm{of} \mathrm{the} \mathrm{ionospheric} \mathrm{ve-}$ locities. Our calculation only shows that, as for the densities, $\mathrm{C}$ III is a good monitor for the F1 region while He II is better for the topside. In between, Fe XV constitutes a good link.

From these considerations, we conclude that the nonredundant set of lines that mostly impact the ionosphere at different altitudes are $\mathrm{H}$ I at $102.572 \mathrm{~nm}, \mathrm{C}$ III at $97.702 \mathrm{~nm}$, $\mathrm{O} \mathrm{V}$ at $62.973 \mathrm{~nm}, \mathrm{He} \mathrm{I}$ at $58.433 \mathrm{~nm}, \mathrm{Fe} \mathrm{XV}$ at $28.415 \mathrm{~nm}$, Si XI at $30.331 \mathrm{~nm}$, and He II at $30.378 \mathrm{~nm}$. Since, however, the impact of Fe XV and Si XI is very similar in terms of electron production and temperature profile, we eliminate the weakest of the two, which is Si XI. As shown in Fig. 2, these discrete lines account for about only half of the electron and ion production.

\section{A different procedure for selecting the best lines}

Our objective now is to find a procedure for selecting those lines that significantly impact the electron density, temperature or productions profiles. This set of lines must be complete enough to allow for the reconstruction of the various profiles that can be generated by the full XUV/EUV spectrum. When several lines give rise to the same profile, however, only one (in general the strongest one or the one that is easiest to measure) should be retained. This is an optimization problem, for which insight can be gained from a so-called connectivity map. We use the technique that was introduced by Dudok de Wit et al. (2005) for reconstructing the solar XUV/EUV spectrum from a small set of lines. Let us consider, for example, the density profile. Two spectral lines are considered to be dissimilar if their impacts on the density profile differ; two profiles are on the contrary similar if the profile changes are the same.

We compute the degree of similarity for parameter $X$ between each pair of lines $\lambda_{1}$ and $\lambda_{2}$ simply by taking the $\mathrm{Eu}-$ clidean distance 


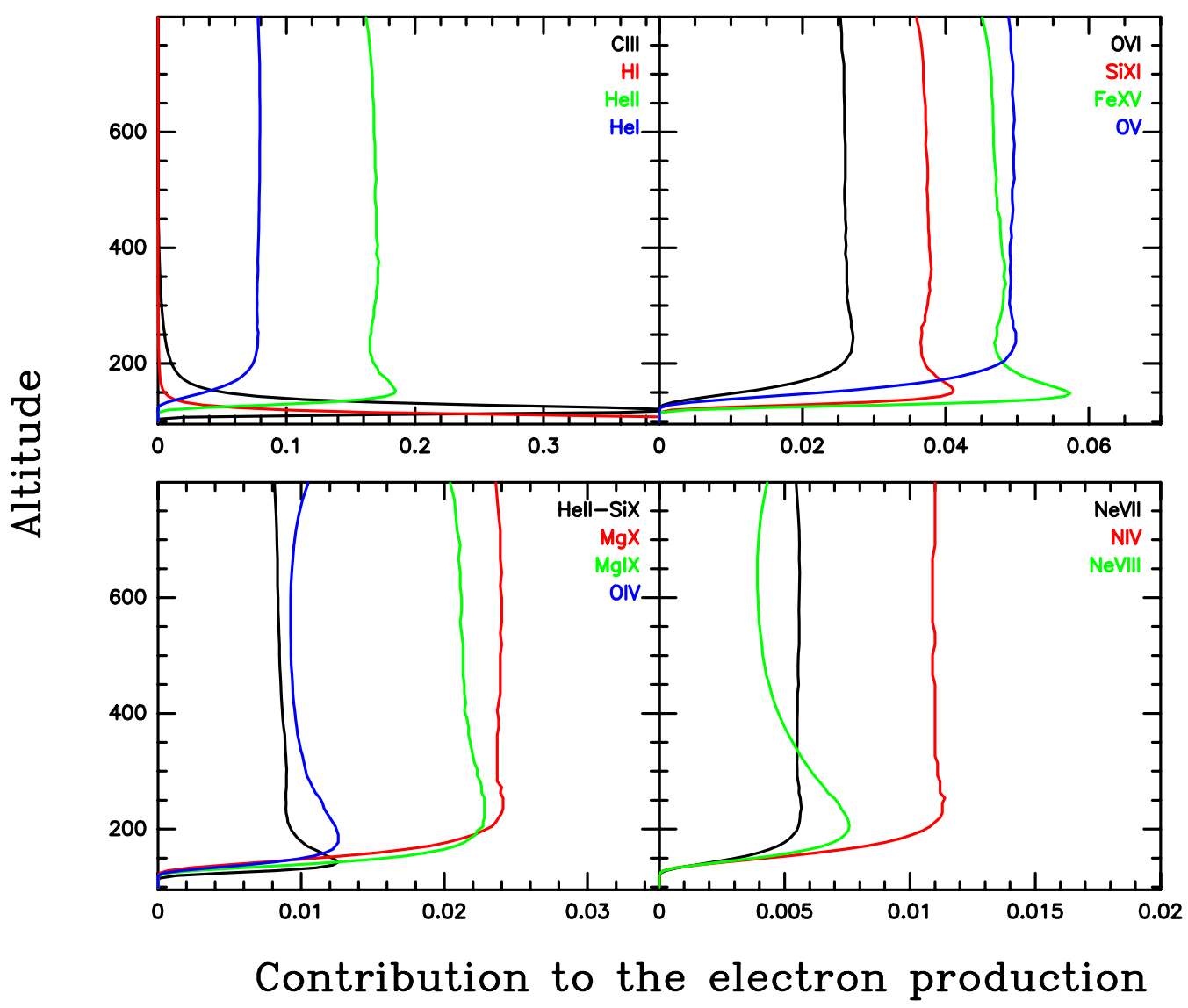

Fig. 4. Relative contribution of each spectral line to the total electron production versus the altitude in $\mathrm{km}$.

$\delta\left(\lambda_{1}, \lambda_{2}\right)=\sqrt{\int\left[X_{\lambda_{1}}(h)-X_{\lambda_{2}}(h)\right]^{2} d h}$,

where $X$ stands either for the relative change in density, temperature or production rate; each parameter is integrated from 100 up to $400 \mathrm{~km}$. We now want to represent the dissimilarity between spectral lines as distances in a low-dimensional space (called connectivity map), in order to make the data accessible to visual inspection and exploration. To do so, we represent each spectral line by a single point in a multidimensional space, in such a way that the distance between each pair of lines equals the distance $\delta\left(\lambda_{1}, \lambda_{2}\right)$ defined above. The closer two lines are, the more similar their impact on the profile of parameter $\mathrm{X}$ is. The dimensionality of this space, in principle, equals 15 , i.e. the number of lines minus one. Since, however, many lines have similar signatures, a projection onto a lower-dimensional space can easily be carried out, while preserving the distances. The appropriate technique for doing such a projection is multidimensional scaling (Borg and Groenen, 1997). In our particular case, the projections in different directions are closely connected to the principal axes of the data (Chatfield and Collins, 1995). Using the Sin-

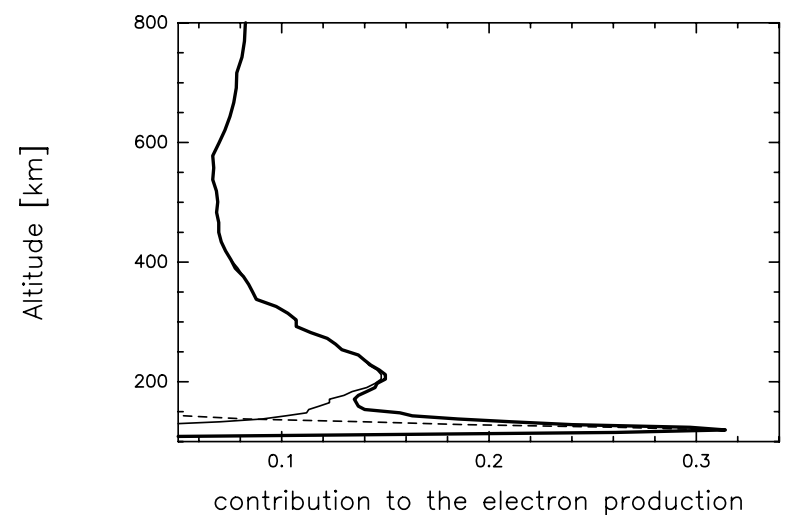

Fig. 5. Linearity check of the impact of the He II and C III lines on the electron production rate. The thick full lines represent the sum of the contributions of He II and C III, the thin line the contribution of He II and the dashed line the contribution of C III.

gular Value Decomposition (Golub and Van Loan, 2000), we express the data as a linear combination of separable modes 


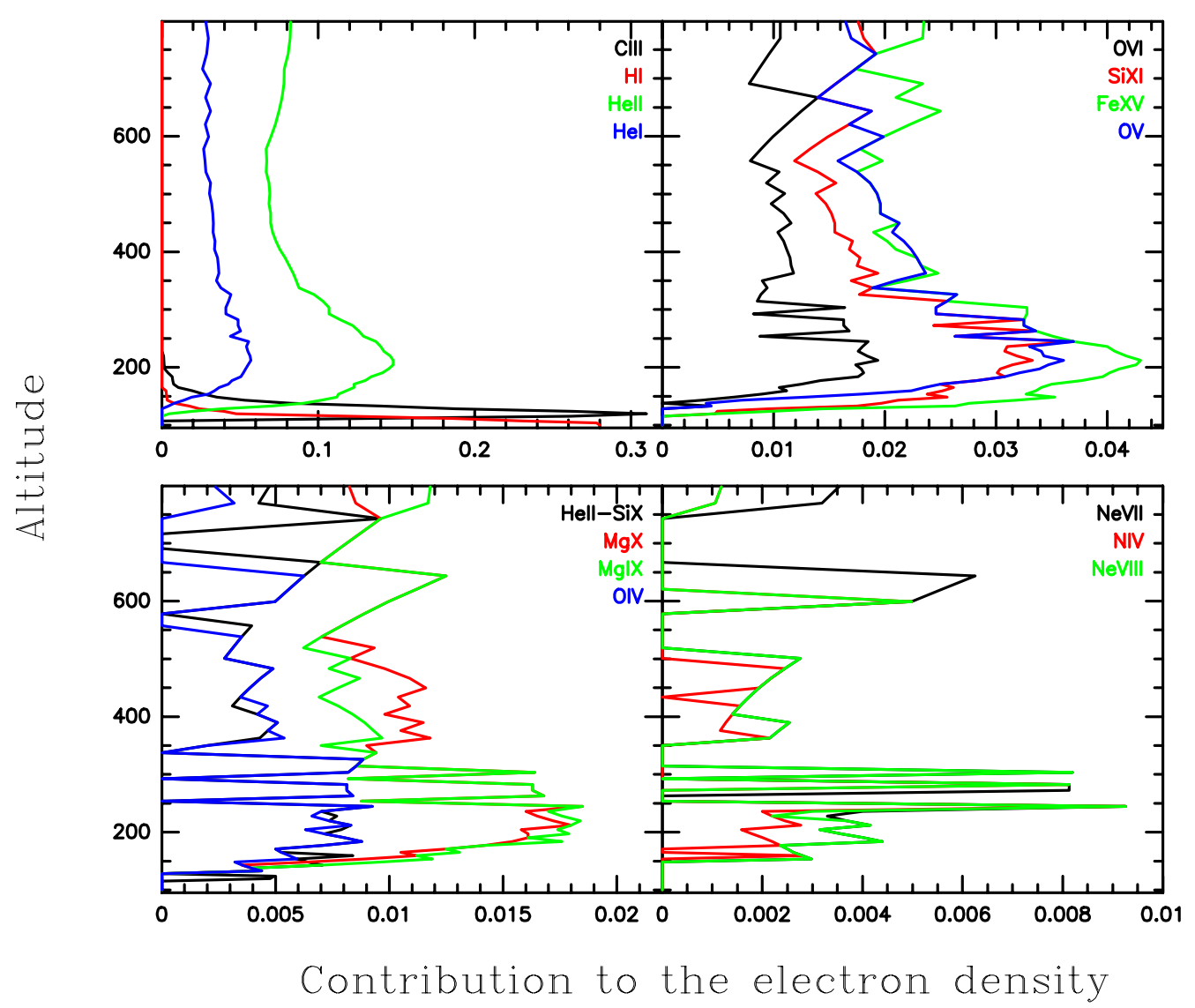

Fig. 6. Relative contribution of the different XUV/EUV lines to the total electron density versus latitude in km. Notice that the scales of the abscissa vary by more than one order of magnitude. Discretization effects show up at very low electron densities.

$X_{\lambda}(h)=\sum_{k=1}^{16} A_{k} f_{k}(h) g_{k}(\lambda)$

with the orthonormality constraint

$$
\left\langle f_{k}(h) f_{l}(h)\right\rangle=\left\langle g_{k}(\lambda) g_{l}(\lambda)\right\rangle=\delta_{k l},
$$

where $\langle\cdots\rangle$ means ensemble averaging. The weights are traditionally sorted in decreasing order $A_{1} \geq A_{2} \geq \ldots \geq A_{16} \geq 0$. The number of modes equals the number of spectral lines. Large weights correspond to modes that describe features shared by many lines. The proportion of variance accounted for by the first $i$ modes is

$V_{i}=\frac{\sum_{k=1}^{i} A_{k}^{2}}{\sum_{k=1}^{16} A_{k}^{2}}$.

In our case, two to three modes are enough to describe over $95 \%$ of the variance. This important result considerably eases the analysis, since it allows us to determine the salient features of the dissimilarity by using a three-dimensional projection. The coordinates of the spectral lines along the $i$-th axis of the connectivity map are given by $A_{i} g_{i}(\lambda)$. Let us stress that the axes of this connectivity do not convey here any clear physical meaning; what matters is the relative distance only between spectral lines.

Figure 8 shows the location of the 16 lines in a threedimensional space, for each of the physical parameters individually, and for all three together. The origin corresponds to the full spectrum, with no lines turned off. Adding a fourth dimension to these plots does not add pertinent information and merely complicates the visualisation.

Each cluster of points in Fig. 8 corresponds to a family of spectral lines that have the same impact, both in terms of amplitude and in terms of profile change. Figure 8a, which deals with density profile changes, reveals the existence of three families of lines. The outstanding H I and the C III lines have a significant impact on the density and moreover lead to different profile changes. Figure 6 indeed shows that the $\mathrm{H}$ I lines affects the density profiles at lower altitudes than the C III line. The other lines are mostly located along a line that goes through the origin, which means that the profile change is the same in shape but not in amplitude. The He II line is the most influential one. The next strongest lines are He I, Fe XV and Si XI. From this we conclude that the best 


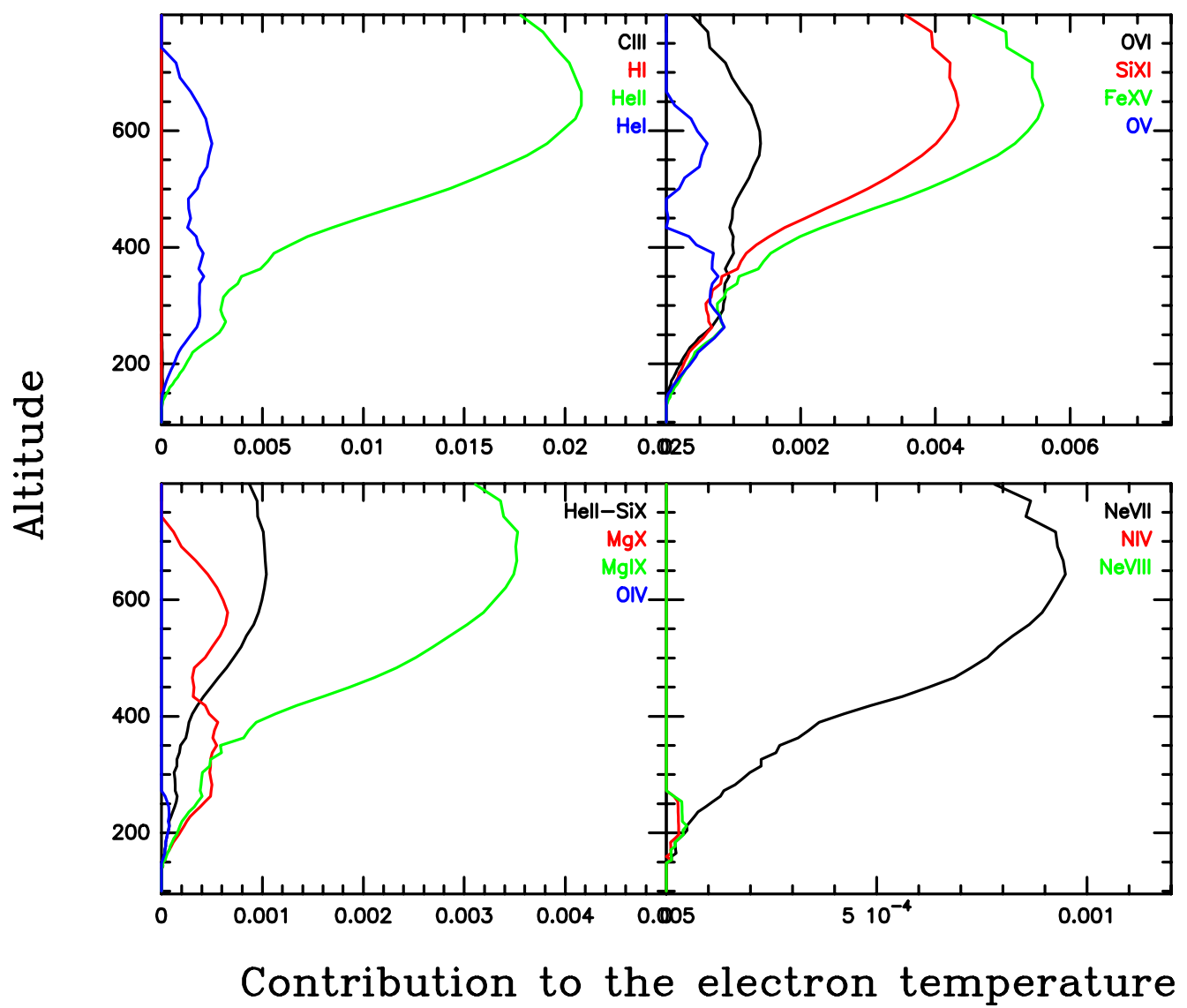

Fig. 7. Relative contribution of the different XUV/EUV lines to the electron temperature. The scales of the abscissa varies from one plot to the other.

candidates for modelling density changes are the H I, C III and He II lines.

Figure $8 \mathrm{~b}$ shows the impact on the temperature profile and reveals a different ordering of the spectral lines, with a prevalence of energetic lines. H I and C III now have little impact, while He II is still as strong, followed by Fe XV, Si XI and $\mathrm{Mg}$ IX. Note also that the lines are essentially distributed in a two-dimensional space, as the third dimension conveys little information. There is indeed more redundancy in temperature profile changes than in density or production rate profiles.

The impact on the production rate (see Fig. 8c) again reveals a different ordering, with four groups of lines. In this figure, we encounter again H I, C III and He II, each of which leads to a different profile. The remaining lines are clustered together and could be represented by Fe XV only. One can finally analyse all three parameters (density, temperature and production rate) together, which gives the plot shown in Fig. 8d. This figure is a weighted average of the previous ones, showing again the predominance of He II, C III, H I, followed by a bunch of other weaker lines such as Fe XV, He I, etc.
Figure 8 emphasizes spectral lines that have a strong impact on the physical parameters. It is also interesting to normalize the profile changes beforehand so that all lines have an equality of weight. We do this by replacing $X_{\lambda}$ by $X_{\lambda} / \sigma_{X}$ prior to the computation of the distances, where $\sigma_{X}$ is the standard deviation of $X_{\lambda}$. The consequence of this normalisation is shown in Fig. 9, in which the impact on all three parameters is considered. The H I and C III lines, which so far behaved differently from the others, still reveal distinct impacts on the ionosphere. The He II line, however, now appears in a cluster that consists of several other lines, such as Fe XV and Mg IX. This result is not surprising, as it means that the relative impact on the ionosphere of all energetic lines is the same. A novel result is the existence of a cluster of lines that was hidden until now, and which consists of lines such as O V, Ne VIII and O III. Although the absolute impact of these lines is weak, the resulting profile change is sufficiently different to take this cluster into consideration in our selection of lines. Our preference goes here to the $\mathrm{O} \mathrm{V}$ line, for it is intense and has been widely used so far in aeronomy. 
a) density

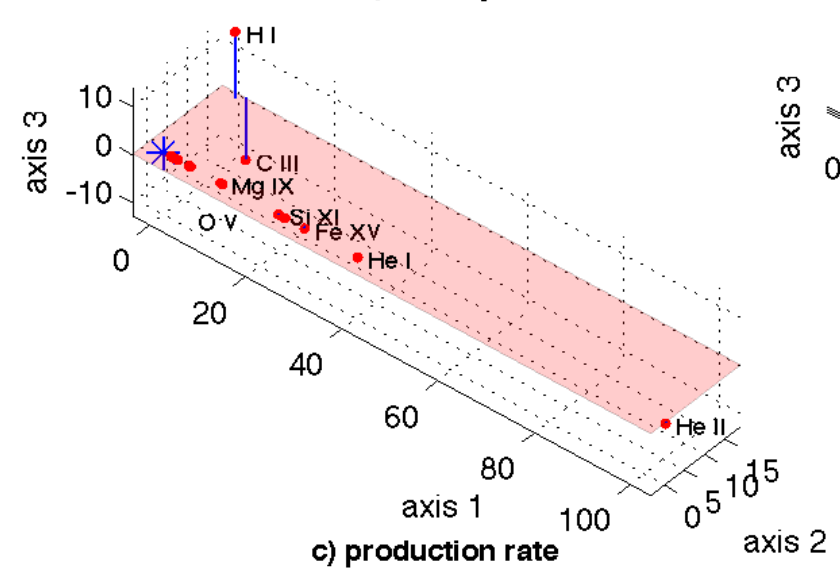

b) temperature

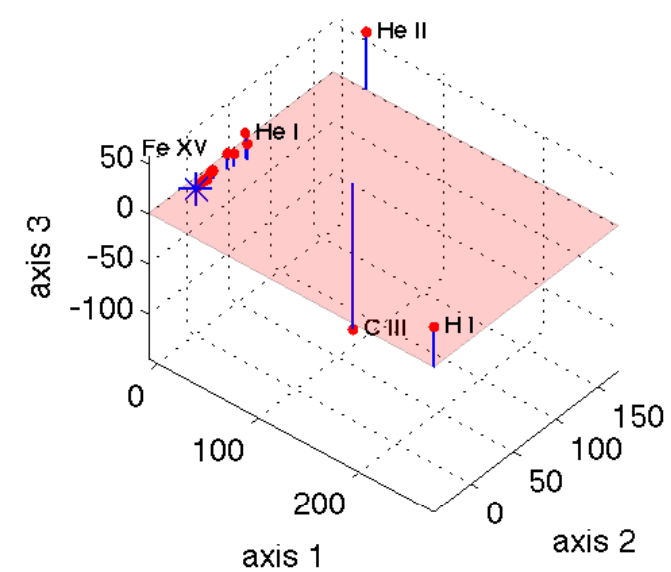

Fig. 8. Three-dimensional connectivity map of the spectral lines, in which the distance between each pair of lines reflects their degree of dissimilarity. The maps correspond to the density profile (a), the temperature profile (b), the production rate (c), and all three parameters together (d). Vertical bars are there merely as a guide to locate the lines with respect to the plane with vertical coordinate $z=0$ (shown in translucent red). Only those lines are labeled, which sufficiently stand out of the clusters of points.

We conclude from Figs. 8 and 9 that the best set of lines for describing the variability of our ionospheric parameters are: H I (102.572 nm), C III (97.702 nm) and He II (30.378 nm), followed by series of less influential lines such as Fe XV $(28.415 \mathrm{~nm}), \mathrm{He} \mathrm{I}(58.433 \mathrm{~nm})$ and $\mathrm{O} \mathrm{V}(62.973 \mathrm{~nm})$. The first three lines are mandatory, while the choice of the latter three could be adapted, for example, to instrumental constraints. We conclude that a dedicated instrument, which continuously measures the absolute irradiance of those 6 lines, would provide all the information that is needed to specify the ionospheric response to the full solar XUV/EUV spectrum. Other inputs have to be added to this list if one aims to model effects such as particle precipitation. Let us stress, however, that in terms of space weather applications, the XUV/EUV spectrum is the prime quantity for ionospheric specification.

Our results apply to stationary regimes. In the case of an eruptive event, the sudden enhancement of the flux results in a transitory regime for which our set of lines may not be opti- mal anymore. Since, however, our set covers regions ranging from the chromosphere up to the hot corona, the spectrum of eruptive phenomena should still be properly covered by it.

\section{Retrieving the full spectrum from these 6 selected lines}

Interestingly, the set of 6 lines we have defined from purely aeronomical considerations is also remarkably good for reconstructing the solar XUV/EUV spectrum. To show this, we use the same approach as in Dudok de Wit et al. (2005) and consider four years of solar XUV/EUV spectra from the TIMED satellite to test the reconstruction. The SEE spectograph on board TIMED routinely measures several spectra per day in the $27-194 \mathrm{~nm}$ range, with an instrumental resolution of $0.4 \mathrm{~nm}$. These data are merged here into a daily measurement from which flares are subtracted. We reconstruct the XUV/EUV spectrum by assuming that the flux in 


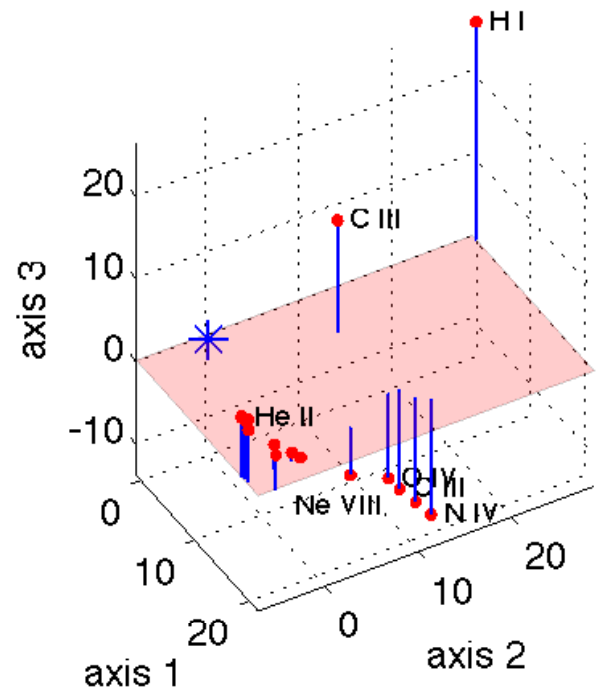

Fig. 9. Same plot as Fig. 8d, after normalizing each quantity to its standard deviation.

each spectral bin between 27 and $194 \mathrm{~nm}$ can be expressed as a linear combination of our 6 spectral lines. The feasibility of such a reconstruction is indeed suggested by the high correlation between irradiances measured at different wavelengths, and has been confirmed by Dudok de Wit et al. (2005).

Here we use one year of TIMED spectra (i.e. the period spanning from February 2002 till February 2003) to estimate the coefficients needed for reconstructing the flux at all wavelengths from our six lines, and use the remaining three years to compare the fitted flux against the true one. Since priority is given to the reconstruction of the spectrally resolved irradiance rather than to the shape of the spectrum, our global error criterion is

$\epsilon_{X}=\frac{\sqrt{\sum_{\lambda} \sum_{t}\left[X(t, \lambda)-X_{\mathrm{fit}}(t, \lambda)\right]^{2}}}{\sqrt{\sum_{\lambda} \sum_{t} X^{2}(t, \lambda)}}$

where $X(t, \lambda)$ is the flux at time $t$ and wavelength $\lambda$.

From our calculation, the relative global error $\epsilon_{X}$ for our set of lines is $6.8 \%$, to be compared with the best combination of 6 lines, which yields $\epsilon_{X}=3.7 \%$. It comes as a surprise that the best set of lines for aeronomy is so close to the best set of lines for reconstructing the XUV/EUV spectrum. The selection criteria are indeed totally different. One possible reason for this agreement is that both approaches lead to sets of lines that issue from different layers of the solar atmosphere. These lines, in addition, are quite evenly distributed in the EUV range of wavelengths, making them good candidates both for 1) representing the dynamics of the solar EUV spectrum at different wavelengths and 2) estimating the impact on the thermosphere/ionosphere system at different altitudes. A direct consequence of this result is the absence of a need for explicitly monitoring the (non-negligible) contri-
Table 1. The six lines that allow one to specify the ionospheric density, temperature and electron production rate.

\begin{tabular}{lll}
\hline Mandatory lines & H I & $102.572 \mathrm{~nm}$ \\
& C III & $97.702 \mathrm{~nm}$ \\
& He II & $30.378 \mathrm{~nm}$ \\
\hline Less influential lines & Fe XV & $28.415 \mathrm{~nm}$ \\
& He I & $58.433 \mathrm{~nm}$ \\
& O V & $62.973 \mathrm{~nm}$ \\
\hline
\end{tabular}

bution of the continuum, as it is already properly taken into account by the spectral lines.

\section{Conclusion}

The objective of this study was to determine whether an ionospheric specification model could use as solar input a small set of spectral lines rather than using the full solar XUV/EUV spectrum. Using TRANSCAR to model the impact of various XUV/EUV lines on three ionospheric parameters (the density profile, the temperature profile and the production rate profile), we find that six lines are sufficient to reproduce the variability of the XUV/EUV spectrum. The solution consists of a selection procedure based on a statistical technique called multidimensional scaling, which allows one to eliminate lines that have a similar impact on the ionosphere in terms of amplitude and profile change.

A direct application of this study is the real-time specification of the ionosphere/thermosphere system. For space weather purposes, it would indeed be much more convenient to have a small dedicated instrument that continuously measures the absolute intensity of a few lines or a few spectral bands, rather than a full-fledged spectograph, which is more appropriate for fundamental science. The LYRA radiometer (Hochedez et al., 2006) on board the PROBA2 satellite, which is due for launch in 2008, will provide experimental ground to our approach. LYRA will continuously measure the EUV/UV spectrum in 4 bands: $1-20 \mathrm{~nm}, 17-70 \mathrm{~nm}$, $115-125 \mathrm{~nm}$ and $200-220 \mathrm{~nm}$. Although the choice of these spectral bands was technology-driven, LYRA will provide the first opportunity to test our spectral reconstruction approach.

Acknowledgements. We thank the COST 724 action for financial and scientific support.

Topical Editor M. Pinnock thanks two anonymous referees for their help in evaluating this paper.

\section{References}

Blelly, P.-L., Lilensten, J., Robineau, A., Fontanari, J., and Alcaydé, D.: Calibration of a numerical ionospheric model with EISCAT observations, Ann. Geophys., 14, 1375-1390, 1996, http://www.ann-geophys.net/14/1375/1996/. 
Blelly, P.-L., Lathuillère, C., Emery, B., Lilensten, J., Fontanari, J., and Alcaydé, D.: An extended TRANSCAR model including ionospheric convection: simulation of EISCAT observations using inputs from AMIE, Ann. Geophys., 23, 419-431, 2005, http://www.ann-geophys.net/23/419/2005/.

Borg, I. and Groenen, P.: Modern Multidimensional Scaling: Theory and Applications, Springer Verlag, Berlin, 1997.

Chatfield, C. and Collins, A. J.: Introduction to Multivariate Analysis, Chapman and Hall, 1995.

Danilov, A. D.: Simplified empirical model of the auroral D region for the international reference ionosphere, Adv. Space Res., 37 , 1038-1044, doi:10.1016/j.asr.2005.01.067, 2006.

Diloy, P.-Y., Robineau, A., Lilensten, J., Blelly, P.-L., and Fontanari, J.: A numerical model of the ionosphere, including the Eregion above EISCAT, Ann. Geophys., 14, 191-200, 1996, http://www.ann-geophys.net/14/191/1996/.

Dudok de Wit, T., Lilensten, J., Aboudarham, J., Amblard, P.-O., and Kretzschmar, M.: Retrieving the solar EUV spectrum from a reduced set of spectral lines, Ann. Geophys., 23, 3055-3069, 2005, http://www.ann-geophys.net/23/3055/2005/.

Floyd, L., Newmark, J., Cook, J., Herring, L., and McMullin, D.: Solar EUV and UV spectral irradiances and solar indices, J. Atmos. Terr. Phys., 67, 3-15, 2005.

Golub, G. H. and Van Loan, C. F.: Matrix Computations, Johns Hopkins Press, Baltimore, 2000.

Hinteregger, H. E.: Representation of Solar EUV Fluxes for Aeronomical Applications, Adv. Space Res., 1, 39-52, 1981.

Hinteregger, H. E. and Katsura, F.: Observational, Reference and Model Data on Solar EUV, from Measurements on AE-E, Geophys. Res. Lett., 8, 1147-1150, 1981.

Hochedez, J.-F., Schmutz, W., Stockman, Y., et al.: LYRA, a solar UV radiometer on Proba2, Adv. Space Res., 37, 303-312, doi: 10.1016/j.asr.2005.10.041, 2006.

Judge, D. L., McMullin, D. R., Ogawa, H. S., Hovestadt, D., Klecker, B., Hilchenbach, M., Mobius, E., Canfield, L. R., Vest, R. E., Watts, R., Tarrio, C., Kuehne, M., and Wurz, P.: First solar EUV irradiances obtained from SOHO by the CELIAS/SEM, Solar Phys., 177, 161-173, 1998.

Kretzschmar, M., Lilensten, J., and Aboudarham, J.: Variability of the EUV quiet Sun emission and reference spectrum using SUMER, Astronomy and Astrophysics, 419, 345-356, doi: 10.1051/0004-6361:20040068, 2004.

Kretzschmar, M., Lilensten, J., and Aboudarham, J.: Retrieving the solar EUV spectral irradiance from the observation of 6 lines, Adv. Space Res., 37, 341-346, doi:10.1016/j.asr.2005.02.029, 2006.

Lathuillère, C. and Menvielle, M.: WINDII thermosphere temperature perturbation for magnetically active situations, J. Geophys. Res., 109, 11 304-+, doi:10.1029/2004JA010526, 2004.

Lilensten, J. and Blelly, P.-L.: Du Soleil à la Terre: aéronomie et métérologie de l'espace, EDP Sciences, Paris, 2000.

Lilensten, J. and Bornarel, J.: Space Weather, Environment and Societies, Springer Verlag, Berlin, 2005.

Lilensten, J., Blelly, P. L., Kofman, W., and Alcaydé, D.: Auroral ionospheric conductivities: a comparison between experiment and modeling, and theoretical f 10.7 -dependent model for EISCAT and ESR, Ann. Geophys., 14, 1297-1304, 1996, http://www.ann-geophys.net/14/1297/1996/.

Lummerzheim, D. and Lilensten, J.: Electron transport and energy degradation in the ionosphere: Evaluation of the numerical solution, comparison with laboratory experiments and auroral observations, Ann. Geophys., 12, 1039-1051, 1994, http://www.ann-geophys.net/12/1039/1994/.

Picone, J. M., Hedin, A. E., Drob, D. P., and Aikin, A. C.: NRLMSISE-00 empirical model of the atmosphere: Statistical comparisons and scientific issues, J. Geophys. Res, 107, 15-1, doi:10.1029/2002JA009430, 2002.

Richards, P. G., Fennelly, J. A., and Torr, D. G.: EUVAC: A solar EUV flux model for aeronomic calculations, J. Geophys. Res., 99, 8981-8992, 1994.

Rottman, G.: The SORCE Mission, Solar Phys., 230, 7-25, doi: 10.1007/s11207-005-8112-6, 2005.

Rozanov, E., Schraner, M., Egorova, T., Ohmura, A., Wild, M., Schmutz, W., and Peter, T.: Solar signal in atmospheric ozone, temperature and dynamics simulated with CCM SOCOL in transient mode, Memorie della Societa Astronomica Italiana, 76, 876-888, 2005.

Schmidtke, G., Woods, T. N., Worden, J., Rottman, G. J., Doll, H., Wita, C., and Solomon, S. C.: Solar EUV irradiance from the San Marco ASSI - A reference spectrum, Geophys. Res. Lett., 19, 2175-2178, 1992.

Schunk, R. W. and Nagy, A. F.: Ionospheres, Cambridge University Press, Cambridge, 2004.

Schwenn, R.: Space Weather: The Solar Perspective, Living Reviews in Solar Physics, 3, 2-31, 2006.

Swartz, W. E. and Nisbet, J. S.: Revised calculations of the F region ambient electron heating by photoelectrons, J. Geophys. Res, 77, 6259-6277, 1972.

Tobiska, W. and Nusinov, A.: ISO 21348 - Process for determining solar irradiances, in: 36th COSPAR Scientific Assembly, vol. 36 of COSPAR, Plenary Meeting, pp. 2621-2462, 2006.

Tobiska, W. K.: Revised solar extreme ultraviolet flux model, J. Atmos. Terr. Phys., 53, 1005-1018, 1991.

Tobiska, W. K. and Eparvier, F. G.: EUV97: Improvements to EUV irradiance modeling in the soft X-rays and FUV, Solar Phys., 177, 147-159, 1998.

Tobiska, W. K., Chakrabarti, S., Schmidtke, G., and Doll, H.: Comparative solar EUV flux for the San Marco ASSI, Adv. Space Res., 13, 255-259, doi:10.1016/0273-1177(93)90022-4, 1993.

Torr, M. R. and Torr, D. J.: Ionization frequencies for major thermospheric constituents as a function of solar cycle 21, Geophys. Res. Lett., 6, 771-774, 1979.

Torr, M. R. and Torr, D. J.: Ionization frequencies for solar cycle 21: revised, J. Geophys. Res., 90, 6675-6678, 1985.

Witasse, O., Lilensten, J., Lathuillère, C., and Blelly, P.-L.: Modeling the OI 630.0 and $557.7 \mathrm{~nm}$ thermospheric dayglow during EISCAT-WINDII coordinated measurements, J. Geophys. Res., 104, 24 639-24 656, 1999.

Woods, T. N., Prinz, D. K., Rottman, G. J., et al.: Validation of the UARS solar ultraviolet irradiances: Comparison with the ATLAS 1 and 2 measurements, J. Geophys. Res., 101, 9541-9570, doi: 10.1029/96JD00225, 1996.

Woods, T. N., Eparvier, F. G., Bailey, S. M., Chamberlin, P. C., Lean, J., Rottman, G. J., Solomon, S. C., Tobiska, W. K., and Woodraska, D. L.: Solar EUV Experiment (SEE): Mission overview and first results, J. Geophys. Res., 110, 1312-1336, 2005. 